\title{
pneumonia
}

Original article

\section{Decreased serum level of thioredoxin 1 in female patients with pneumonia and its combinational use with haptoglobin for the specific diagnoses of pneumonia and lung cancer}

\author{
Mee-Kyung Cha, II-Han Kim \\ Department of Life Science and Technology, Pai Chai University, Daejeon, Republic of Korea
}

Corresponding author: II-Han Kim, Department of Life Science and Technology, Pai Chai University, 11-3 Techno 1ro Yuseong-Gu, Daejeon, 305-509, Republic of Korea, Tel: 82-42-520-5379, Email: ihkim@pcu.ac.kr

Author contributions: All authors met ICMJE authorship criteria. MC conducted the analysis and acquisition/interpretation of data. IK designed the research plan and writing of the manuscript. IK and MC critically reviewed the manuscript for important intellectual content. IK and $\mathrm{MC}$ agree with the manuscript results and conclusions

Received 13 December 2014; Accepted 1 April 2015; Published 29 June 2015

Citation: Cha MK, Kim IH. Decreased serum level of thioredoxin 1 in female patients with pneumonia and its combinational use with haptoglobin for the specific diagnoses of pneumonia and lung cancer. pneumonia 2015;6:18-25

\begin{abstract}
Thioredoxin $1(\operatorname{Tr} x 1)$ and haptoglobin $(\mathrm{Hp})$ are known to be involved in pathophysiology. This study was conducted to evaluate their diagnostic significance. We employed an enzyme-linked immunosorbent assay (ELISA) to determine the concentrations of both Trx 1 and $\mathrm{Hp}$ in sera from female patients with community-acquired pneumonia (CAP) and those with lung cancer. The Trx1 levels remarkably decreased in cases of female patients with CAP, while the $\mathrm{Hp}$ levels increased in both female patients with lung cancer and CAP. In addition, the serum levels of Trx1 were not significantly changed in patients with lung cancer, rheumatoid arthritis, and cardiovascular diseases compared to healthy controls. At the cut-off point of 0.396 at $A_{450 \mathrm{~nm}}$ on the receiver operating characteristic (ROC) curve, Trx1 could discriminate between patients with CAP from normal female controls with a sensitivity of $72.5 \%$, a specificity of $89.8 \%$, and area under the ROC curve (AUC) of $0.877 \pm 0.040$. The serum levels of Trx 1 in female CAP patients were inversely correlated with the levels of $\mathrm{Hp}(p<0.05)$. The characteristic reduction in serum Trx1 levels, especially in female CAP patients, indicates that Trx1 could be used as a diagnostic marker for CAP. The advantage of serum Trx1 over $\mathrm{Hp}$ in discriminating female CAP patients among female patients who have a positive serum level of Hp suggests the use of Trx1 as an excellent combination marker with $\mathrm{Hp}$ for the specific diagnosis of CAP and lung carcinoma, because serum $\mathrm{Hp}$ levels increase in female patients with lung cancer and those with CAP without selectivity.
\end{abstract}

Keywords: pneumonia, lung cancer, thioredoxin 1, haptoglobin, companion marker 


\section{Introduction}

The 12-kDa human cytosolic thioredoxin 1 (Trx1) contains a redox-active dithiol moiety in its conserved active-site sequence (-Cys-Gly-Pro-Cys-) [1]. In resting cells, Trx1 resides in the cytosol, but activation by a wide variety of stimuli leads to its translocation to the nucleus or the secretion of this protein [2,3]. Trx1 catalyses thioldisulfide exchange reactions via the two cysteine residues [1]. The exchange reaction activity is involved in various biological activities, including the regulation of the activity of transcription factors, such as nuclear factor (NF-KB) and activator protein-1 [2,4]. Moreover, reduced Trx1 inhibits apoptosis by binding to apoptosis signaling kinase $[1,5]$. The extracellular Trx1 acts as a chemokine and co-cytokine, stimulating cytokine secretion and cell proliferation [6,7]. Due to these biological activities, Trx1's involvement in redox processes could be important in the pathophysiology of cancer and inflammation [5-8].

Haptoglobin (Hp) protein, a plasma glycoprotein, is synthesised mainly in hepatocytes, dermal cells, pulmonary cells, and renal cells and released into the blood. In blood plasma, $\mathrm{Hp}$ binds free haemoglobin released from erythrocytes and prevents the loss of iron, and thereby inhibits haemoglobin's oxidative activity [9]. $\mathrm{Hp}$ is also known as a positive acute-phase protein with a plasma level that rapidly increases in response to any infection or inflammatory process such as communityacquired pneumonia (CAP) [10-12]. In addition, elevated levels of $\mathrm{Hp}$ have been demonstrated in various cancers, including lung cancer [13-15].

CAP is the most common potentially fatal infectious disease worldwide [16]. Despite advances in prevention strategies, such as antibiotic treatment, significant improvement in the mortality rate is still lacking. This high mortality is mainly due to the absence of an effective diagnostic marker for CAP, which delays the timing of adequate antibiotic therapy. Because CAP is an infectious disease, commonly used laboratory values include the white blood cell count, C-reactive protein, and procalcitonin. In recent years, biomarkers have been intensively studied in CAP, not only for the correct diagnosis of CAP but also with respect to diagnosing its microbiological aetiology, the severity of disease, prognosis, and treatment decisions [17].

In the present study, we observed that the serum Trx1 levels are remarkably decreased in female patients with CAP compared to healthy female controls. We also demonstrated that the Hp level was significantly increased in serum from patients with CAP and those with lung cancer. The characteristic decrease of serum Trx1 levels, especially in female CAP patients, suggested that Trx 1 could be useful as a diagnostic marker for CAP. Taken together, these findings indicated that Trx 1 itself is a potential marker for CAP and is also an effective companion marker to $\mathrm{Hp}$ for the diagnoses of CAP and lung cancer.

\section{Methods}

\subsection{Patients and sample collection}

All sera of normal persons (control) and patients with CAP and lung cancer were obtained from Caucasians, White. The sera and the clinical information for the study participants were provided from Asterand Bioscience (http://www.asterandbio.com) and BioServe (http://www. bioserve.com) and are summarised in Table 1 and Table 2. All samples were collected from their collaborating clinical sites with full adherence to proper informed consent, as well as their strict institutional review board and Health Insurance Portability and Accountability Act compliance. To make them suitable for a biomarker study, all the sera were collected and treated according to the instructions of the Food and Drug Administration and the National Cancer Institute. All serum samples were taken prior to surgery and/or the commencement of chemotherapeutic medications. Samples were provided from subjects who were determined to have cardiovascular disease if they met the New York Heart Association criteria [18]; however, if the information for classification was not available, other diagnostic criteria of cardiovascular disease determined by echocardiogram, coronary angiogram, or high troponin levels following a cardiac episode were also included. Samples were provided from subjects with rheumatoid arthritis who met the American College of Rheumatology criteria for rheumatoid arthritis [19] and who were rheumatoid factor positive. Normal control serum samples were provided from subjects who were healthy and had no known clinical conditions (Eric M. Langlois, BioServe, Personal Communication, 31 March 2015).

\subsection{Enzyme-linked immunosorbent assay (ELISA) for serum Trx1 and Hp protein levels}

An Express $^{\mathrm{TM}}$ ELISA kit (rabbit), an indirect ELISA kit from GenScript Inc. (USA), was used to measure serum protein levels as per the manufacturer's instructions. Antibodies to human Trx1 and Hp proteins were obtained by injecting purified proteins into rabbits to form antisera and then purifying the antiserum on a Protein A column. In brief, the sample dilution buffer containing an appropriate volume of human serum ( $5 \mu$ for Trx1 determination; $10 \mu \mathrm{l}$ for $\mathrm{Hp}$ determination) was coated onto a 96-well ELISA plate for 30 minutes, followed by the addition of the respective rabbit polyclonal antibodies (primary antibodies) against the antigens ( $\operatorname{Trx} 1$ or $\mathrm{Hp}$ ). After the primary antibody was allowed to react with the coated antigen for 1 hour, the antigen-antibody complex was treated with a horseradish peroxidase (HRP)-conjugated secondary antibody, followed by immobilisation for 1 hour and then washing 3 times with the washing solution. TMB $\left(3,33^{\prime}, 5\right.$, $5^{\prime}$-tetramethylbenzidine) was used as the substrate, and a sulfuric acid solution ( $2 \mathrm{M}$ sulfuric acid) was used to stop the enzymatic reaction. Protein levels were determined by measuring the absorbance at $450 \mathrm{~nm}$ in triplicate; mean values of 3 measurements are given. The intra-assay precision (coefficient of variations [CV]) for analysis of Trx1 
Table 1 Characteristics of normal control subjects and non-cancer groups

\begin{tabular}{lllll}
\hline Characteristics & No. of samples & Age (years) mean \pm SD & BMI mean \pm SD (range) & No. Smoking (\%) \\
\hline $\begin{array}{l}\text { Normal control: Caucasian, } \\
\text { White }\end{array}$ & 100 & $44.1 \pm 14.8$ & $27.1 \pm 4.6(18.6-42.9)$ & $54(54.0)$ \\
$\quad$ Male & 50 & $44.5 \pm 14.9$ & $27.5 \pm 4.2(18.6-38.2)$ & $23(46.0)$ \\
$\quad$ Female & 50 & $44.2 \pm 14.6$ & $26.6 \pm 4.9(19.5-42.9)$ & $31(62.0)$ \\
CAP $\quad 80$ & $67.0 \pm 16.8$ & $27.0 \pm 5.9(16.4-47.2)$ & $56(70.0)$ \\
$\quad$ Male & 40 & $67.4 \pm 16.6$ & $25.8 \pm 4.5(16.4-37.9)$ & $34(85.0)$ \\
$\quad$ Female & 40 & $66.7 \pm 17.2$ & $28.2 \pm 6.8(18.4-47.2)$ & $22(55.0)$ \\
Rheumatoid arthritis & 30 & & $28.9 \pm 5.5(17.2-42.2)$ & $15(50.0)$ \\
$\quad$ Female & & $59.5 \pm 13.1$ & $30.8 \pm 6.9(18.8-51.1)$ & $16(53.3)$ \\
Cardiovascular disease & 30 & & & \\
$\quad$ Female & & $65.0 \pm 11.3$ & & \\
\hline
\end{tabular}

CAP, community-acquired pneumonia; BMI, body mass index; SD, standard deviation of the mean

Table 2 Characteristics of serum samples from patients with lung cancer

\begin{tabular}{ll}
\hline Characteristics & $\begin{array}{l}\text { No. of } \\
\text { samples }\end{array}$ \\
\hline Non-small cell lung cancer & $111(65.4$ \\
& $\pm 10.4)^{\mathrm{a}}$ \\
Male & $50(65.8$ \\
& $\pm 10.2)$ \\
Female & $61(61.8$ \\
& $\pm 10.3)$ \\
Non-small cell lung cancer stage (sub-stage) & \\
Stage I (IA 19, IB 20) & 39 \\
Stage II (IIA 16, IIB 16) & 32 \\
Stage III (IIIA 21, IIIB 9) & 30 \\
Stage IV & 10 \\
Non-small cell lung cancer sub-type & \\
Adenocarcinoma, NOS & $29(19)^{\mathrm{b}}$ \\
Adenocarcinoma with mixed subtypes & $17(12)$ \\
Mixed cell adenocarcinoma & $6(3)$ \\
Papillary adenocarcinoma, NOS & $2(1)$ \\
Acinar cell carcinoma & $7(5)$ \\
Bronchiolo-alveolar adenocarcinoma, NOS & $9(2)$ \\
Bronchiolo-alveolar carcinoma, non-mucinous & $1(1)$ \\
Mucinous adenocarcinoma & $5(4)$ \\
Squamous cell carcinoma, NOS & $19(6)$ \\
Squamous cell carcinoma, keratinising, NOS & $3(2)$ \\
Adenosquamous carcinoma & $3(2)$ \\
Carcinoid tumor, NOS & $2(1)$ \\
Neuroendocrine carcinoma & $2(0)$ \\
N/A & $6(3)$ \\
\hline
\end{tabular}

There are no data on other tumor markers from the lung cancer samples.

NOS, not otherwise specified; N/A, not available

${ }^{\text {a }}$ Mean age $\pm S D$, years; ${ }^{b}$ Number of female patients and $\mathrm{Hp}$ were $5.77 \pm 2.49 \%$ (mean \pm SD) and $2.57 \pm 1.23 \%$, respectively. The interassay CV\% was $9.18 \pm 3.53 \%$ and $5.97 \pm 3.97 \%$, respectively, based on a sample of 30 female patients with CAP.

\subsection{Statistical analysis}

GraphPad Prism version 5.04 for Windows (GraphPad Software, USA) and MedCalc statistical software version 12.4.0.0 (MedCalc Software bvba, Belgium) were used for statistical analyses. We used the Pearson product-moment correlation coefficient to test for associations between different variables. The $t$ tests and one-way analysis of variance (ANOVA) tests were performed to calculate the $p$ value. The $p$ value was considered to be statistically significant if $p<0.05$. Testing for normality of the data was performed using the D'Agostino-Pearson normality test.

\section{Results}

\subsection{Hp levels in lung cancer and CAP patients}

A potential difference in serum $\mathrm{Hp}$ levels between lung cancer and CAP patients was examined. In detail, serum $\mathrm{Hp}$ levels were measured in 111 lung cancer patients distributed over a wide range of ages (age distribution: 41 to 85 years) and 80 CAP patients (age distribution: 41 to 85 years) and subjected to receiver operating characteristic (ROC) curve plotting. Concentration measurements are graphically shown in Figure 1 and statistically summarised in Table 3. Area under the ROC curve (AUC) values were calculated by the partial integration of the ROC curve; sensitivity and specificity values are summarised in Table 4.

As seen in Figure 1 and Table 3 , the mean $\mathrm{Hp}$ concentrations in the normal male/female control (NMF) 


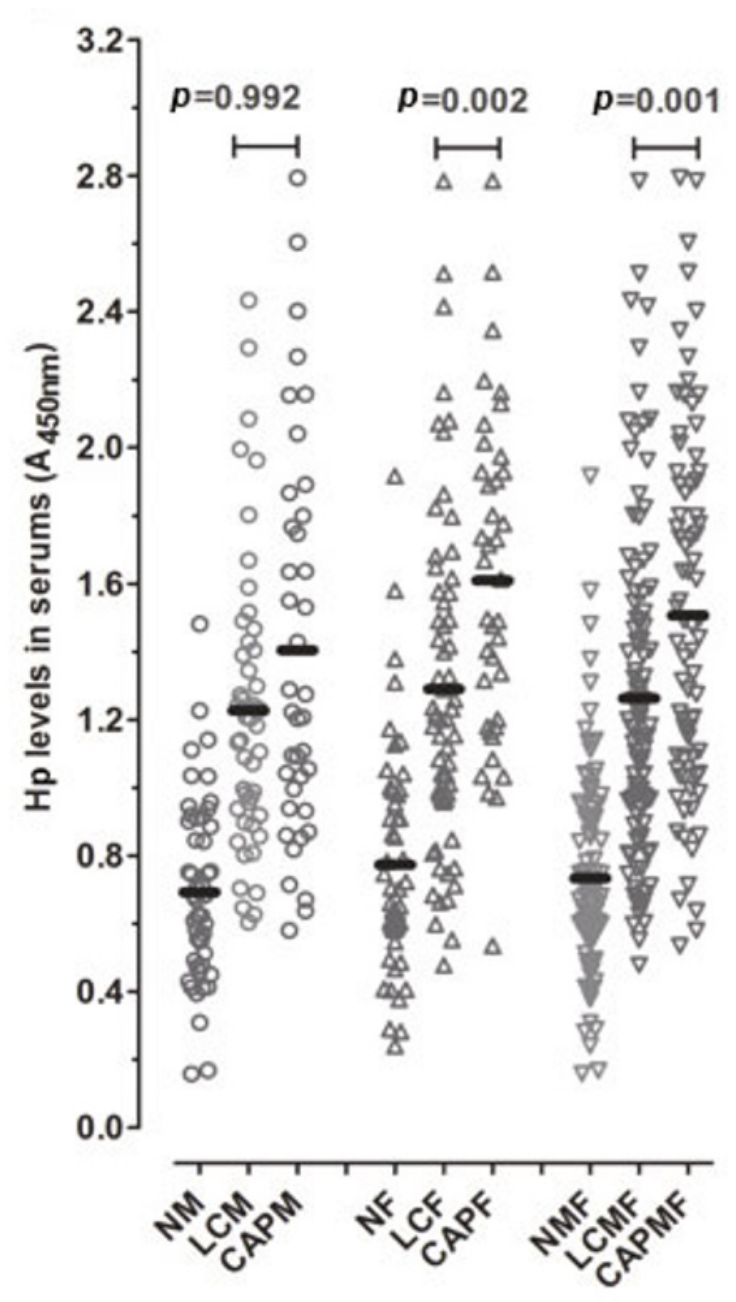

NM, normal male control; LCM, lung cancer male; CAPM, CAP male; NF, normal female control; LCF, lung cancer female; CAPF, CAP female; NMF, normal male/female control; LCMF, lung cancer male/female; CAPMF, CAP male/female

Figure 1 Serum haptoglobin ( $\mathrm{Hp}$ ) levels in the lung cancer and community-acquired pneumonia (CAP) groups. Clinicopathological information for each patient was provided by the supplier. The individual mean value $(n=3)$ is depicted as a scatter plot. The median value of each group is depicted by a horizontal line. The statistic values are shown in Table 3. group, the lung cancer male/female (LCMF) group, and the CAP male/female (CAPMF) group were $0.733,1.26$, and 1.51 , respectively. This shows that the serum $\mathrm{Hp}$ levels increased by approximately $72 \%$ and $106 \%$ in LCMF and CAPMF patients, respectively, compared to NMF subjects, indicating that the $\mathrm{Hp}$ level is approximately $20 \%$ higher in CAP patients than in lung cancer patients. The difference in the elevation of $\mathrm{Hp}$ levels in the lung cancer versus CAP groups was more profound in the female groups (LCF versus CAPF: 25\%) than that in the male groups (LCM versus CAPM: 14\%). As indicated in Figure 1, the difference in the serum $\mathrm{Hp}$ level between male patients with lung cancer and CAP is not statistically significant $(p>0.05)$, whereas the difference between female patients is evident $(p=0.002)$.

For the LCM and CAPM groups compared to the NM subjects, the AUC values of $\mathrm{Hp}$ were measured to be, respectively, 0.878 and 0.865 , with a sensitivity of $75.5 \%$ and $72.5 \%$ and a specificity of $84.0 \%$ and $88.0 \%$ (Table 4 ). For the LCF and CAPF groups compared to the NF subjects, AUC values of $\mathrm{Hp}$ were, respectively, 0.820 and 0.905 , with a sensitivity of $78.7 \%$ and $82.5 \%$ and a specificity of $73.5 \%$ and $89.8 \%$.

These data show that $\mathrm{Hp}$ can be used to screen for lung cancer and CAP, as displayed in Table 4 (AUC values for LCMF: 0.860, those for CAPMF: 0.882), although Hp has a disadvantage in its selectivity due to simultaneous elevations of $\mathrm{Hp}$ levels in both patient groups. It is worth noting that the selectivity between female lung cancer and CAP patients is superior compared to male patients.

\subsection{Trx1 levels in patients with CAP and various diseases}

We then examined the difference in serum Trx1 levels between patients with CAP and those with lung cancer. We aimed to investigate serum Trx1 levels due to reports that the intracellular synthesis of Trx1 increases in response to cancerous processes [5]. Serum Trx1 levels may also be increased in response to lung carcinoma progression. We selected lung cancer as a representative cancer for this

Table 3 Statistics for the serum haptogobulin ( $\mathrm{Hp}$ ) levels of normal control subjects, lung cancer, and community-acquired pneumonia (CAP) patients

\begin{tabular}{llllllllll}
\hline & NM & LCM & CAPM & NF & LCF & CAPF & NMF & LCMF & CAPMF \\
\hline Number of values & 50 & 50 & 40 & 50 & 61 & 40 & 100 & 111 & 78 \\
25\% percentile & 0.488 & 0.928 & 0.939 & 0.585 & 0.965 & 1.18 & 0.546 & 0.958 & 1.08 \\
Median & 0.676 & 1.20 & 1.22 & 0.702 & 1.20 & 1.61 & 0.682 & 1.20 & 1.46 \\
75\% percentile & 0.904 & 1.42 & 1.80 & 0.984 & 1.58 & 1.93 & 0.914 & 1.49 & 1.90 \\
Mean & 0.693 & 1.23 & 1.40 & 0.774 & 1.29 & 1.61 & 0.733 & 1.26 & 1.51 \\
Std. deviation & 0.267 & 0.421 & 0.575 & 0.337 & 0.502 & 0.482 & 0.305 & 0.467 & 0.537 \\
Std. error & 0.038 & 0.060 & 0.092 & 0.048 & 0.064 & 0.077 & 0.031 & 0.045 & 0.061 \\
\hline
\end{tabular}

NM, normal male control; LCM, lung cancer male; CAPM, CAP male; NF, normal female control; LCF, lung cancer female; CAPF, CAP female; NMF, normal male/female control; LCMF, lung cancer male/female; CAPMF, CAP male/female 



NM, normal male control; CAPM, CAP male; NF, normal female control; CAPF, CAP female; NMF, normal male/female control; CAPMF, CAP male/female; Arth F, rheumatoid arthritis disease female; Cardio F, cardiovascular disease female; LCMF, lung cancer male/ female; LC, lung cancer patients stage I, II, III, and IV

Figure 2 Serum thioredoxin 1 (Trx1) levels in the community-acquired pneumonia (CAP), rheumatoid arthritis, cardiovascular, and lung cancer groups. Changes of serum Trx1 levels along with lung cancer progression are depicted in panel B. The individual mean value $(n=3)$ is depicted as a scatter dot plot with the median value of each group (horizontal line). The statistic values are shown in Table 3. The $p$ value shown in the upper portion of panel B was obtained from one-way ANOVA, and the other $p$ values were obtained from t-tests.

Table 4 Parameters from the ROC analysis on the serum haptoglobin ( $\mathrm{Hp}$ ) levels in lung cancer and community-acquired pneumonia (CAP) patients

\begin{tabular}{|c|c|c|c|c|c|c|}
\hline & \multicolumn{3}{|l|}{ Lung cancer } & \multicolumn{3}{|l|}{ CAP } \\
\hline & $\overline{\mathrm{LCM}}$ & LCF & LCMF & CAPM & CAPF & CAPMF \\
\hline$\overline{\mathrm{AUC} \pm \mathrm{SE}^{\mathrm{a}}}$ & $0.878 \pm 0.033$ & $0.820 \pm 0.040$ & $0.860 \pm 0.028$ & $0.865 \pm 0.040$ & $0.905 \pm 0.037$ & $0.882 \pm 0.027$ \\
\hline Sensitivity (\%) & 75.5 & 78.7 & 76.6 & 72.5 & 82.5 & 81.2 \\
\hline Specificity (\%) & 84.0 & 73.5 & 79.8 & 88.0 & 89.8 & 83.8 \\
\hline Cut-off value $\left(A_{450 \mathrm{~nm}}\right)$ & 0.938 & 0.954 & 0.954 & 0.958 & 1.136 & 0.995 \\
\hline
\end{tabular}

Hp measurements of the normal male and female controls used as the reference

LCM, lung cancer male; LCF, lung cancer female; LCMF, lung cancer male/female; CAPM, CAP male; CAPF, CAP female; CAPMF, CAP male/female; AUC, area under the ROC curve; SE, standard error of the mean ${ }^{\mathrm{a}} p<0.0001$ 
study because CAP is an inflammatory disease of the lung. Serum Trx1 levels were measured by ELISA in the patients, as well as in normal male and female controls.

As seen in Figure 2, the mean concentrations of Trx1 in the NMF, CAPMF, and LCMF groups were $0.504,0.417$, and 0.568 , respectively, showing that the serum levels of Trx1 decreased by approximately $21 \%$ in the CAPMF patients. Trx1 levels increased by approximately $13 \%$ in the LCMF group compared to the NMF group. In addition, Figure 2B shows that serum Trx1 levels increased along with lung cancer progression, indicating that the increase of Trx1 levels may be caused by the cancerous process. The data for NF, NM, LCM, and LCF subjects are not separately shown here because of their similarity with the data for the NMF and LCMF groups.

Interestingly, serum Trx1 levels were at remarkably lower values in CAPF patients (0.360) than in CAPM patients (0.457), although the Trx1 levels are nearly the same in the respective controls. This finding indicated that the serum levels of Trx1 decrease by approximately $27 \%$ and $8 \%$ in CAPF and CAPM patients, respectively, compared to their corresponding normal controls (NF: 0.490; NM: 0.495) (Figure 2A).

Serum Trx1 levels were also measured in female patients with rheumatoid arthritis (Arth F) and cardiovascular disease (Cardio F). We chose to look at these groups because the intracellular synthesis of $\operatorname{Trx} 1$ is increased in response to oxidative stress, so we reasoned that serum Trx1 levels may also be increased in response to inflammatory and oxidative stress-induced diseases. As seen in Figure 2A, the mean serum Trx 1 concentrations in the Arth $F$ and Cardio $F$ groups were 0.485 and 0.552 , respectively, indicating that Trx1 levels in the Arth $\mathrm{F}$ patients are nearly the same as the levels in the NF group $(p=0.761)$. In contrast, the Trx1 levels increased by approximately $3 \%$ in Cardio $\mathrm{F}$ patients compared to the NF group $(p=0.001)$. The opposite trends observed for the serum Trx1 levels between CAP and the other investigated diseases support the utility of Trx1 as a diagnostic marker for CAP over $\mathrm{Hp}$ because serum $\mathrm{Hp}$ levels are significantly elevated in both patients with CAP and those with lung cancer.

Taken together, the data described above indicate that Trx1 may be useful as a promising CAP marker, especially for female subjects, because it is capable of clearly identifying CAP patients, whether they have been affected by cancer or another disease.

To clarify this result, ROC curve analysis was performed to compare the Trx1 levels of the CAP group and the lung cancer group, which was used as a representative cancerous disease of the same organ, with the Trx1 measurements of the normal male and female controls. For the CAPM and CAPF groups versus the NMF group, the AUC values for Trx1 were measured to be, respectively, 0.638 and 0.877 with a sensitivity of $65.0 \%$ and $72.5 \%$ and a specificity of $64.0 \%$ and $89.8 \%$, respectively (Table 5 ). For the LCMF group compared to the NMF group, the AUC value of Trx1 was measured to be 0.636 , with a sensitivity of $62.2 \%$ and a specificity of $64.0 \%$.

In addition, ROC curve analysis was performed for the Trx1 levels of the cardiovascular group, with the increased Trx1 values compared to normal female values. For the Cardio F group compared to the NF group, the AUC value of Trx1 was measured to be $0.677 \pm 0.063$, indicating that this type of oxidative stress-related disease apparently increases Trx1 levels.

Taken together, the results described above indicate that Trx1 can discriminate between the female CAP group and the normal female controls at a probability of about as high as $88 \%$ and can be used as a CAP marker with superior sensitivity and specificity.

\subsection{Reverse correlation of Trx1 levels with Hp levels}

As is understood from the data, $\mathrm{Hp}$ is a good marker for both male and female patients with CAP, whereas Trx1 exhibits a function similar to $\mathrm{Hp}$ in female patients. The characteristic difference between $\mathrm{Hp}$ and $\operatorname{Tr} \mathrm{1}$ is that the CAP group has a higher serum $\mathrm{Hp}$ level but a lower serum Trx1 level compared to the corresponding normal controls.

Table 5 Parameters from the ROC analysis on the serum thioredoxin 1 (Trx1) levels in patients with lung cancer and communityacquired pneumonia (CAP)

\begin{tabular}{|c|c|c|c|c|}
\hline & \multicolumn{3}{|l|}{ CAP } & \multirow{2}{*}{$\begin{array}{l}\text { Lung cancer } \\
\text { LCMF }\end{array}$} \\
\hline & $\overline{\text { CAPM }}$ & CAPF & CAPMF & \\
\hline$\overline{\mathrm{AUC} \pm S \mathrm{SE}^{\mathrm{a}}}$ & $0.638 \pm 0.062$ & $0.877 \pm 0.040$ & $0.756 \pm 0.039$ & $0.636 \pm 0.038$ \\
\hline Sensitivity (\%) & 65.0 & 72.5 & 51.2 & 62.2 \\
\hline Specificity (\%) & 64.0 & 89.8 & 92.9 & 64.0 \\
\hline Cut-off value $\left(A_{450 \mathrm{~nm}}\right)$ & 0.479 & 0.396 & 0.397 & 0.532 \\
\hline
\end{tabular}

Trx1 measurements of the normal male and female controls used as the reference

CAPM, CAP male; CAPF, CAP female; CAPMF, CAP male/female; LCMF, lung cancer male/female; AUC, area under the ROC curve; SE, standard error of the mean

${ }^{\mathrm{a}} p<0.000$ 


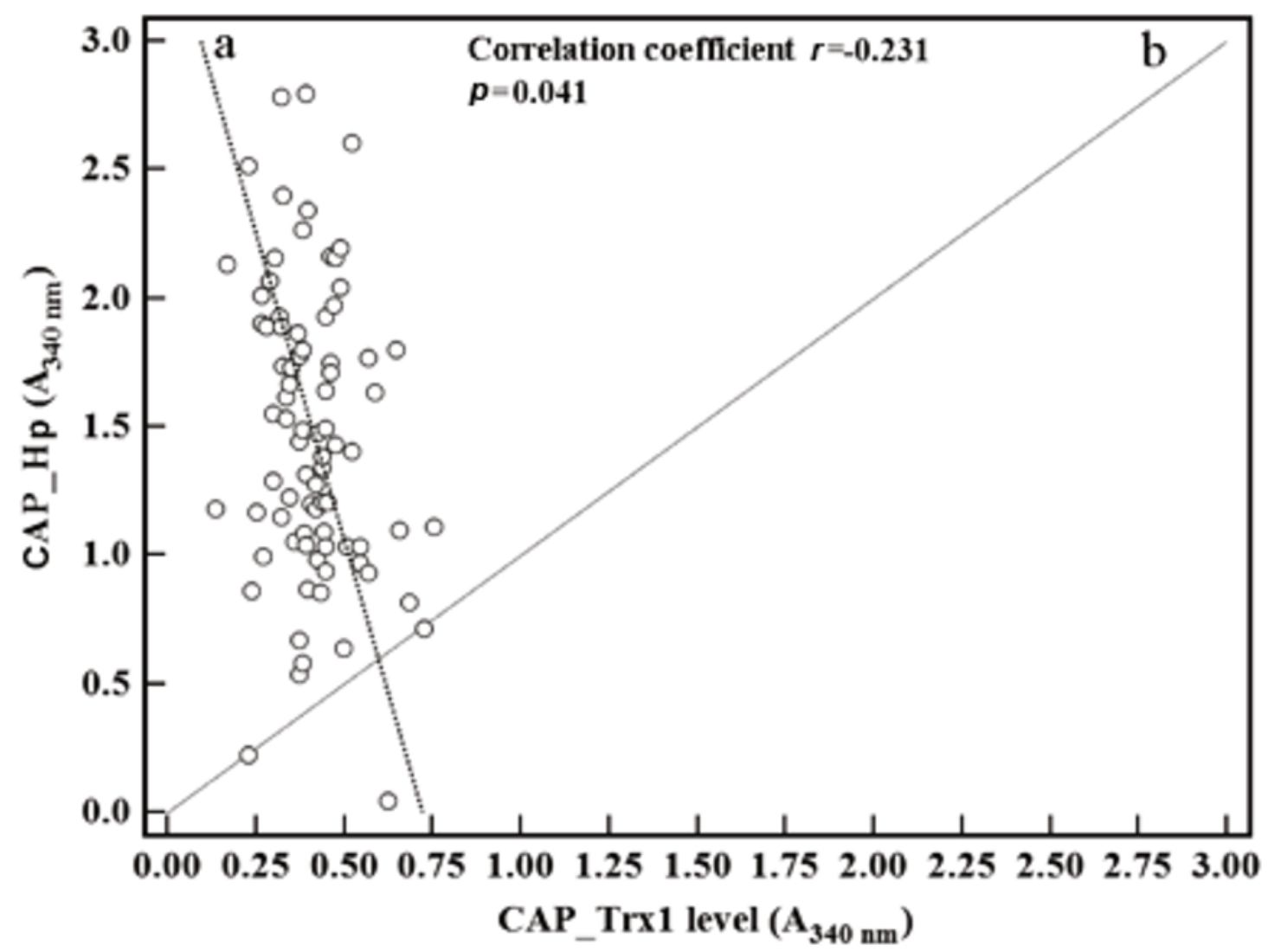

$r$, Pearson correlation coefficient constant from Pearson correlation analysis; $p$ value obtained from the correlation analysis

Figure 3 Correlation between the serum thioredoxin 1 (Trx1) and haptoglobin ( $\mathrm{Hp}$ ) levels in community-acquired pneumonia (CAP) patients. The individual mean serum levels $(n=3)$ in 80 patients with CAP ( $n=40$ for female and $n=40$ for male) were depicted as a scatter diagram. The individual serum levels of Trx1 were displayed along the $\mathrm{x}$-axis, and those of $\mathrm{Hp}$ in the corresponding patients are plotted along the $y$-axis. Lines " $a$ " and " $b$ " are reduced major axis line and line of equality, respectively.

To confirm these contrary results, the correlations between the Trx1 measurements and the $\mathrm{Hp}$ measurements of the CAP group were analysed, and the results are shown in Figure 3. The Trx1 measurements were found to be inversely proportional to the $\mathrm{Hp}$ measurements, ( $r=-0.231, p=0.041)$, as seen in Figure 3. For testing for normality of the data, the D'Agostino-Pearson normality test was performed. The data sets for Trx1 and Hp levels were normally distributed ( $\operatorname{Tr} x 1: p=0.06$; Hp: $p=0.30$ ).

\section{Discussion}

This study showed that Trx1 can be used as a diagnostic marker for CAP and an effective companion diagnostic marker to $\mathrm{Hp}$ for lung cancer. Trx1 was found to be present in significantly lower concentrations in the serum of female patients with CAP than in the blood of normal females. In addition, the serum Trx1 levels in patients with cardiovascular diseases and lung cancer were measured to be significantly higher than those in normal controls. Furthermore, the serum level of Trx1 was observed to increase along with lung cancer progression. In contrast to Trx1, much higher serum levels of $\mathrm{Hp}$ were detected in CAP patients, as well as lung cancer patients without selectivity, compared to normal controls. The Trx1 measurements were found to be inversely proportional to the Hp measurements. Overall, the specifically remarkable decrease of Trx1 levels in female CAP patients suggests that the reduction may be caused by a female CAP-specific action and not by general oxidative stress and inflammatory actions. Trx1 was reported to be elevated in patients with various carcinomas and inflammatory diseases [8,12], which is consistent with our results showing significant increases of serum Trx1 levels in patients with lung cancer and cardiovascular disease.

On the basis of the results summarised above, $\operatorname{Trx} 1$ may be useful as a diagnostic marker for screening female patients with CAP; female CAP patients are characterised as having a lower serum Trx1 level compared to the controls. Monitoring Trx1 levels in the blood plasma may be useful for the diagnosis and follow-up of CAP. The serum level of Trx1 significantly decreases in the female patients compared to female controls with excellent specificity $(89.8 \%)$ and selectivity (AUC $=0.877$ ) for the female patients, regardless of whether they have been affected by cancer or non-cancer diseases. Trx 1 has an advantage over $\mathrm{Hp}$ in selecting female patients with CAP because serum $\mathrm{Hp}$ levels increase in both female patients with lung cancer and CAP without specificity. Consequently, Trx1 can be used not only as a potential CAP marker in females but is also as an excellent companion diagnostic marker with $\mathrm{Hp}$ for discriminating CAP patients from females with high serum levels of $\mathrm{Hp}$. 
Considering a high serum level of $\mathrm{Hp}$ in both female patients with lung cancer or CAP and a significant decrease of serum Trx1 level in female patients with CAP, but the increasing tendency of serum Trx1 level as a function of the progress of lung carcinoma in the female patients, taken together, these observations suggested that serum Trx1 can be used as not only a novel pneumonia biomarker applicable to female patients, but also as a combinational marker with $\mathrm{Hp}$ for the specific diagnosis of female patients with lung carcinoma.

Trx1 serves not only as a reactive oxygen species detoxifier but also as a regulator of signal transduction pathways and peroxide responses [1,2]. Thus, a decreased serum level of Trx1 in CAP patients, especially in the female patients, remains unresolved. In addition to extracellular levels of Trx1 and $\mathrm{Hp}$, it could be interesting to determine their intracellular levels in the paired tissues. Given that the reduction of the serum Trx1 level in CAP patients is not female-specific, the detailed in vivo function of serum Trx1 in CAP patients is worth further investigation. In fact under certain conditions, the expression of Trx1 or the secretion of Trx1 into extracellular fluid is either negatively or positively regulated, which can be explained by differences regarding cell types, cell conditions, or strength of stimulation.

\section{Acknowledgments}

MK Cha performed this work during her research sabbatical given by Pai Chai University, Republic of Korea. The authors would like to thank Mr. Byung-Joon Park, Pai Chai University, Republic of Korea, for his technical support.

Funding: The authors have no funding to report.

Competing interests: All authors declare no competing interests.

Copyright: This is an open-access article distributed under the terms of the Creative Commons Attribution License, which permits unrestricted use, distribution, and reproduction in any medium, provided the original author and source are credited.

\section{References}

1. Arnér ES, Holmgren A. Physiological functions of thioredoxin and thioredoxin reductase. Eur J Biochem 2000;267:61029.http://dx.doi.org/10.1046/j.1432-1327.2000.01701.x PMID:11012661

2. Nakamura H, Nakamura K, Yodoi J. Redox regulation of cellular activation. Annu Rev Immunol 1997;15:351-69. http://dx.doi.org/10.1146/annurev.immunol.15.1.351 PMID:9143692

3. Rubartelli A, Bajetto A, Allavena G, Wollman E, Sitia R. Secretion of thioredoxin by normal and neoplastic cells through a leaderless secretory pathway. J Biol Chem 1992;267:24161-4. PMID:1332947

4. Holmgren A. Thioredoxin. Annu Rev Biochem 1985;54:237-71.http://dx.doi.org/10.1146/annurev. bi.54.070185.001321 PMID:3896121
5. Saitoh M, Nishitoh H, Fujii M, Takeda K, Tobiume K, Sawada $Y$ et al. Mammalian thioredoxin is a direct inhibitor of apoptosis signal-regulating kinase (ASK) 1. EMBO J 1998;17:2596-606.http://dx.doi.org/10.1093/ emboj/17.9.2596 PMID:9564042

6. Rosen A, Lundman P, Carlsson M, Bhavani K, Srinivasa BR, Kjellström $\mathrm{G}$ et al. A CD4+ T cell line-secreted factor, growth promoting for normal and leukemic B cells, identified as thioredoxin. Int Immunol 1995;7:625-33.http://dx.doi. org/10.1093/intimm/7.4.625 PMID:7547690

7. Schenk $H$, Vogt M, Dröge W, Schulze-Osthoff K. Thioredoxin as a potent costimulus of cytokine expression. J Immunol 1996;156:765-71. PMID:8543831

8. Cha MK, Suh $\mathrm{KH}, \mathrm{Kim} \mathrm{IH}$. Overexpression of peroxiredoxin I and thioredoxin1 in human breast carcinoma. J Exp Clin Cancer Res 2009;28:93.http://dx.doi.org/10.1186/17569966-28-93 PMID:19566940

9. Wassell J. Haptoglobin: function and polymorphism. Clin Lab 2000;46:547-52. PMID:11109501

10. Langlois MR, Delanghe JR. Biological and clinical significance of haptoglobin polymorphism in humans. Clin Chem 1996;42:1589-600. PMID:8855140</jrn>

11. Sadrzadeh SM, Bozorgmehr J. Haptoglobin phenotypes in health and disorders. Am J Clin Pathol 2004;121 Suppl:S97-104. PMID:15298155

12. $\mathrm{Xu} \mathrm{J}, \mathrm{Li} \mathrm{T}, \mathrm{Wu} \mathrm{H}, \mathrm{Xu}$ T. Role of thioredoxin in lung disease. Pulm Pharmacol Ther 2012;25:154-62.http://dx.doi. org/10.1016/j.pupt.2012.01.002 PMID:22293327

13. Beckman G, Eklund A, Fröhlander N, Stjernberg N. Haptoglobin groups and lung cancer. Hum Hered 1986;36:258-60.http://dx.doi.org/10.1159/000153638 PMID:3759102

14. Awadallah SM, Atoum MF. Haptoglobin polymorphism in breast cancer patients form Jordan. Clin Chim Acta 2004;341:17-21.http://dx.doi.org/10.1016/j. cccn.2003.10.032 PMID:14967153

15. Zhao C, Annamalai L, Guo C, Kothandaraman N, Koh SC, Zhang $\mathrm{H}$ et al. Circulating haptoglobin is an independent prognostic factor in the sera of patients with epithelial ovarian cancer. Neoplasia 2007;9:1-7.http://dx.doi. org/10.1593/neo.06619 PMID:17325738

16. Welte T, Suttorp N, Marre R. CAPNETZ-communityacquired pneumonia competence network. Infection 2004;32:234-8.http://dx.doi.org/10.1007/s15010-0043107-z PMID:15293080

17. Christ-Crain M, Müller B. Biomarkers in respiratory tract infections: diagnostic guides to antibiotic prescription, prognostic markers and mediators. Eur Respir J 2007;30:55673.http://dx.doi.org/10.1183/09031936.00166106 PMID:17766633

18. Classes of heart failure. 2015 [cited 2015 Mar 31]; Available from: http://www.heart.org/HEARTORG/Conditions/ HeartFailure/AboutHeartFailure/Classes-of-Heart-Failure_ UCM_306328_Article.jsp

19. Arnett FC, Edworthy SM, Bloch DA, McShane DJ, Fries JF, Cooper NS et al. The American Rheumatism Association 1987 revised criteria for the classification of rheumatoid arthritis. Arthritis Rheum 1988;31:315-24.http://dx.doi. org/10.1002/art.1780310302 PMID:3358796 